victims affected by this oil are known as the 'Yusho I' cases. A similar 'Yusho II' event occurred in Taiwan in 1979.

It is currently standard practice to assess exposure to chlorophenols by measuring the levels of these chemicals in urine. Rappe, however, suggests that blood measurement of the PCDF and PCDD contaminants present in the chlorinated phenols is a superior method. Urine concentrations of chlorinated phenols fall rapidly to undetectable levels in a very short time whereas PCDDs and PCDFs can be detected in blood years after exposure. Although the significance of TCDD levels in blood is not yet clear, many believe that it is important to have more of these measurements to try and relate blood levels to clinical symptoms.

It is a pity this information is not available now for assessing the carcinogenic risk from TCDD. Reports from Sweden (Hardell and Sandstrom Br. J. Cancer 39, $711 ; 1979)$ suggest that forestry workers exposed to chlorinated phenols and the herbicide 2,4,5-T have a sixfold higher incidence of soft tissue sarcomas. A repeat of the investigation by one of the Swedish scientists to take account of some criticisms of the earlier study confirms the initial findings (Hardell Scand. J. Work envir. Hlth 7, 119; 1981).

However, studies conducted in other countries have so far failed to confirm Hardell's reports. A cohort of herbicide applicators in Finland exposed to phenoxy herbicides for at least two weeks between 1955 and 1971 have been monitored since 1972. The incidence of tumours in this group is no higher than would be expected and no cases of soft tissue sarcoma have been observed. Similarly, preliminary findings from a study in New Zealand, reported by A.H. Smith (Wellington Hospital), in which soft tissue sarcoma cases were compared with patients with other forms of cancer showed that the individual's place of work bore no relationship to the type of tumour seen.

The different findings from the various studies are disturbing. So much so that a workshop study group on the health implications of TCDD exposure concluded that it was a matter of urgency that additional studies be done to determine whether there is indeed a causal relationship between soft tissue sarcoma and exposure to phenoxy herbicides such as 2,4,5-T.

Experimental design of the epidemiological studies may have some bearing on the different results obtained. If so, it is important that new studies should take account of this. There is general agreement that all the studies performed to assess the carcinogenic risk from exposure to TCDD and phenoxy herbicides were well designed. However, it is felt that some studies may have bias which has so far gone unrecognized. If this is the case it will be some time yet before there is unequivocal evidence about the health risk from exposure to $2,4,5-\mathrm{T}$.

\title{
Parasites affect behaviour of mice
}

from F.E.G. Cox

PARASITIC WORMS are ubiquitous in small mammals, even in many laboratory colonies, and are almost invariably ignored in behavioural studies. Two recent investigations indicate, however, that they may markedly influence both the acquisition of dominance and general exploratory behaviour of mice.

When male mice are reared in isolation and then put together fighting occurs and a hierarchy of dominance is established with one dominant male. This simple procedure has allowed an Australian scientist, W.J. Freeland, to investigate the effects of nematode infections on the behaviour of mice ${ }^{1}$. Using groups of two infected and one uninfected mice, he found that those given 50 or 150 larvae of Nematospiroides dubius became dominant as often as uninfected ones but those given 250 larvae became dominant less frequently than would be expected. Once dominance in uninfected mice was established, however, a subsequent infection with 250 larvae did not lead to its loss. Freeland argues that, as dominance may be related to mating success, infected mice are at a reproductive disadvantage and that females mating with uninfected or lightly infected males protect themselves and their offspring from infection and also select males with genes for resistance to infection.

Even though these results are convincing and the conclusions self-evident, they are subject to a number of reservations. First, the number of mice used in each trial, three, was small, although 20-30 trials were used for each level of larval infestation; a more satisfactory procedure would have been to use $6-10$ mice. Second, the numbers of worms that brought about behavioural changes were relatively large. In natural infections the majority of mice carry small numbers of worms and very few carry large numbers ${ }^{2}$ and 250 is more than would normally be expected. The reasoning also fails to take into account the true nature of nematode infections in mice in the wild for the female is as likely to become infected from another male as from her mate as there is no permanent pair-bonding and, as rodent territories are not absolute, males tend to roam over large areas.

The suggestion that female mice might effectively select for genes for resistance is also open to question. Although the importance of genetic factors in resistance to nematodes in general and to N. dubius in particular is well documented $^{3}$, individual animals acquire their initial infections with large and small numbers of worms purely by the

F.E.G. Cox is Professor of Zoology at King's College, London. chance ingestion of infective stages and only later mount an immune response; so in primary infections, like the ones described, the female would be selecting for luck rather than resistance. Furthermore, more active males range further and thus have more opportunities to become infected; this is well known for ectoparasites such as ticks ${ }^{4}$ and is presumably also true for worms of various kinds.

The second set of experiments were carried out in the United States at the State University of New York and Cornell ${ }^{5}$. In these experiments, mice were infected with the dog nematode Toxocora canis. The larvae of this worm cause considerable damage by burrowing about the body of abnormal hosts and may cause blindness in children. In mice, the larvae may enter the nervous system and brain. The American workers infected mice with $T$. canis and subjected them to a number of behavioural tests to assess such activities as exploratory behaviour, areas traversed, avoidance reactions and spatial discrimination in mazes. The various tests were carried out sequentially over a period of 115 days and in all tests infected mice did less well than infected ones. The authors conclude that $T$. canis infection reduces exploratory activity, learning and motor coordination. These results are easier to understand than those of the dominance experiments for the mice infected with $T$. canis were all found to have parasites in the brain at autopsy, but a direct correlation between brain damage and behavioural changes has not yet been established.

These two sets of experiments taken together, however, do suggest that parasites can modify the behaviour of rodents and although this is well known in other hosts the gross behavioural changes are usually brought about by physical damage such as is caused when a parasite lodges in the eye or brain or occupies a considerable amount of the body cavity. The interactions between parasite and host may, however, be more subtle than this and parasites in the gut or kidney may affect the odours produced by the host or may even affect its hormone balance. It is likely that parasites will be increasingly implicated in alterations in host behaviour; however the nature of these changes will not be resolved by the use of simple behavioural experiments but will have to follow the advances being made in our understanding of the underlying mechanisms of animal behaviour.

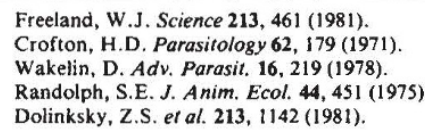

